\title{
STABILIZATION OF HYBRID SYSTEMS BY FEEDBACK CONTROL BASED ON DISCRETE-TIME STATE AND MODE OBSERVATIONS ${ }^{\dagger}$
}

\author{
Jianqiu $\mathrm{Lu}^{1 *}$, Yuyuan $\mathrm{Li}^{2}$, Xuerong Mao${ }^{1}$, Qinwei Qiu²
}

\begin{abstract}
Recently, Mao [1] proposed a kind of feedback control based on discretetime state observations to stabilize continuous-time hybrid stochastic systems in mean-square sense. We find that the feedback control there still depends on the continuous-time observations of the mode. However, it usually costs to identify the current mode of the system in practice. So we can further improve the control to reduce the control cost by identifying the mode at discrete times when we make observations for the state. In this paper, we aim to design such a type of feedback controls based on the discrete-time observations of both state and mode to stabilize the given unstable hybrid stochastic differential equations (SDEs) in the sense of mean-square exponential stability. Moreover, a numerical example is given to illustrate our results.
\end{abstract}

Key Words: Brownian motion, Markov chain, mean-square exponential stability, discrete-time feedback control.

\section{INTRODUCTION}

Hybrid stochastic differential equations (SDEs) (also known as SDEs with Markovian switching), usually used to model practical systems where they may experience abrupt changes in their structure and parameters, have been attracting a lot of attention in recent years. Particularly, as the most fundamental problem in engineering, the asymptotic stability has been studied extensively $[2,3,4,6,12,13,14,18,20$, $21,22,23,24,26,27,28,29,30]$. Here we mention that $[10,11]$ are two of the most cited papers while [17] is the first book in this area.

One classical topic in this field is the problem of stabilization, i.e. designing a control function $u(x(t), r(t), t)$ which usually appears in the drift part

\footnotetext{
*Manuscript received 11 February 2016 and accepted on 8 February 2017.

*The authors are with ${ }^{1}$ Department of Mathematics and Statistics, University of Strathclyde, Glasgow G1 1XH, U.K.

${ }^{2}$ College of Information Sciences and Technology, Donghua Univerisity, Shanghai 201620, China.

* Jianqiu Lu (corresponding author, e-mail: jianqiu.lu@strath.ac.uk).
}

such that the controlled system

$$
\begin{aligned}
d x(t) & =[f(x(t), r(t), t)+u(x(t), r(t), t)] d t \\
& +g(x(t), r(t), t) d w(t)
\end{aligned}
$$

will be stable though the original system (1.1) with $u(x(t), r(t), t)=0$ is unstable, where $t \geq 0, r(t)$ is a Markov chain, $x(t) \in R^{n}$ is the state, $w(t)=$ $\left(w_{1}(t), \cdots, w_{m}(t)\right)^{T}$ is an $m$-dimensional Brownian motion and the SDE is in the Itô sense.

Wang et al. in [25] designed a state feedback controller to stabilize bilinear uncertain time-delay stochastic systems with Markovian jumping parameters in mean square sense. In [5], the problem of almost sure exponential stabilization of stochastic systems by statefeedback controls was discussed. A robust delayedstate-feedback controller that exponentially stabilizes uncertain stochastic systems was proposed in [15]. It is observed that the state feedback controllers in these papers require continuous observations of the system state $x(t)$ for all time $t \geq t_{0}$. Recently, Mao [1] first proposed to design a discrete-time feedback control $u\left(x\left(\delta\left(t, t_{0}, \tau\right)\right), r(t), t\right)$ in order to make the controlled 
system

$$
\begin{aligned}
d x(t) & =\left[f(x(t), r(t), t)+u\left(x\left(\delta\left(t, t_{0}, \tau\right)\right), r(t), t\right)\right] d t \\
& +g(x(t), r(t), t) d w(t)
\end{aligned}
$$

become exponentially stable in mean square. Here $\tau>$ 0 is a constant and

$$
\delta\left(t, t_{0}, \tau\right)=t_{0}+\left[\left(t-t_{0}\right) / \tau\right] \tau
$$

in which $\left[\left(t-t_{0}\right) / \tau\right]$ is the integer part of $\left(t-t_{0}\right) / \tau$. The advantage of such a discrete-time feedback control is that it requires only state observations $x\left(t_{0}+k \tau\right)$ at discrete times $t_{0}, t_{0}+\tau, t_{0}+2 \tau, \cdots$ and hence it will cost much less and more realistic. Despite this advantage, we can take a further step to make it even better. We observe that the feedback control in Mao [1] is based on the discrete-time observations of the state $x\left(t_{0}+k \tau\right)(k=0,1,2, \cdots)$ but still depends on the continuous-time observations of the mode $r(t)$ on $t \geq t_{0}$. This is perfectly fine if the mode of the system can be fully observed at no cost. However, it usually costs to identify the current mode of the system in practice. So we can further improve the control to reduce the control cost by identifying the mode at discrete times when we make observations for the state. Therefore, in this paper, we will consider an $n$ dimensional controlled hybrid system

$$
\begin{aligned}
d x(t)=[ & f(x(t), r(t), t) \\
& +u\left(x\left(\delta\left(t, t_{0}, \tau\right)\right), r\left(\delta\left(t, t_{0}, \tau\right), t\right)\right] d t \\
& +g(x(t), r(t), t) d w(t)
\end{aligned}
$$

on $t \geq t_{0}$, where our new feedback control is based on the discrete observations of state $x\left(t_{0}+k \tau\right)$ and mode $r\left(t_{0}+k \tau\right)$.

Due to the difficulties arisen from the discretetime Markov chain $r\left(t_{0}+k \tau\right)$, the analysis in this paper will be much more complicated in comparison with the related previous papers and new techniques will be developed. Our main results will be formed in Section 3 and Sections 4 after giving preliminaries in Section 2. We will discuss an example in Section 5 to verify the effectiveness of the results and conclude our paper in Section 6.

\section{Notation and Problem Statement}

In this paper, we use the following notation. Let $\left(\Omega, \mathcal{F},\left\{\mathcal{F}_{t}\right\}_{t \geq 0}, \mathbb{P}\right)$ be a complete probability space with a filtration $\left\{\mathcal{F}_{t}\right\}_{t \geq 0}$ satisfying the usual conditions (i.e. it is increasing and right continuous with $\mathcal{F}_{0}$ contains all $\mathbb{P}$-null sets). Let $w(t)=\left(w_{1}(t), \cdots, w_{m}(t)\right)^{T}$ be an $m$-dimensional Brownian motion defined on the probability space. If $A$ is a vector or matrix, its transpose is denoted by $A^{T}$. If $x \in R^{n}$, then $|x|$ is its Euclidean norm. If $A$ is a matrix, we let $|A|=$ $\sqrt{\operatorname{trace}\left(A^{T} A\right)}$ be its trace norm and $\|A\|=\max \{|A x|$ : $|x|=1\}$ be the operator norm. If $A$ is a symmetric matrix $\left(A=A^{T}\right)$, denote by $\lambda_{\min }(A)$ and $\lambda_{\max }(A)$ its smallest and largest eigenvalue, respectively. By $A \leq 0$ and $A<0$, we mean $A$ is non-positive and negative definite, respectively. Denote by $L_{\mathcal{F}_{t}}^{2}\left(R^{n}\right)$ the family of all $\mathcal{F}_{t}$-measurable $R^{n}$-valued random variables $\xi$ such that $\mathbb{E}|\xi|^{2}<\infty$, where $\mathbb{E}$ is the expectation with respect to the probability measure $\mathbb{P}$.

Let $r(t), t \geq 0$, be a right-continuous Markov chain on the probability space taking values in a finite state space $S=\{1,2, \cdots, N\}$ with generator $\Gamma=\left(\gamma_{i j}\right)_{N \times N}$ given by

$$
\begin{aligned}
& \mathbb{P}\{r(t+\Delta)=j \mid r(t)=i\} \\
& = \begin{cases}\gamma_{i j} \Delta+o(\Delta) & \text { if } i \neq j, \\
1+\gamma_{i i} \Delta+o(\Delta) & \text { if } i=j,\end{cases}
\end{aligned}
$$

where $\Delta>0$. Here $\gamma_{i j} \geq 0$ is the transition rate from $i$ to $j$ if $i \neq j$ while

$$
\gamma_{i i}=-\sum_{j \neq i} \gamma_{i j}
$$

We assume that the Markov chain $r(\cdot)$ is independent of the Brownian motion $w(\cdot)$. It is known that almost all sample paths of $r(t)$ are piecewise constant except for a finite number of simple jumps in any finite subinterval of $R_{+}(:=[0, \infty))$. We stress that almost all sample paths of $r(t)$ are right continuous.

Consider an $n$-dimensional uncontrolled unstable linear hybrid SDE

$$
d x(t)=A(r(t)) x(t) d t+\sum_{k=1}^{m} B_{k}(r(t)) x(t) d w_{k}(t)
$$

on $t \geq 0$, with initial data $x(0)=x_{0} \in L_{\mathcal{F}_{0}}^{2}\left(R^{n}\right)$. Here $A, B_{k}: S \rightarrow R^{n \times n}$ and we will often write $A(i)=$ $A_{i}$ and $B_{k}(i)=B_{k i}$. Now we are required to design a feedback control $u(x(\delta(t)), r(\delta(t)))$ based on the discrete-time state and mode observations in the drift part so that the controlled linear SDE

$$
\begin{aligned}
d x(t) & =[A(r(t)) x(t)+u(x(\delta(t)), r(\delta(t)))] d t \\
& +\sum_{k=1}^{m} B_{k}(r(t)) x(t) d w_{k}(t)
\end{aligned}
$$


will be mean-square exponentially stable, where $u$ is a mapping from $R^{n} \times S$ to $R^{n}, \tau>0$ and

$$
\delta(t)=[t / \tau] \tau \quad \text { for } t \geq 0,
$$

in which $[t / \tau]$ is the integer part of $t / \tau$. As the given $\operatorname{SDE}$ (2.1) is linear, it is natural to use a linear feedback control. One of the most common linear feedback controls is the structure control of the form $u(x, i)=$ $F(i) G(i) x$, where $F$ and $G$ are mappings from $S$ to $R^{n \times l}$ and $R^{l \times n}$, respectively, and one of them is given while the other needs to be designed. These two cases are known as:

- State feedback: design $F(\cdot)$ when $G(\cdot)$ is given;

- Output injection: design $G(\cdot)$ when $F(\cdot)$ is given.

Again, we will often write $F(i)=F_{i}$ and $G(i)=G_{i}$. Then the controlled system (2.2) becomes

$$
\begin{aligned}
d x(t) & =[A(r(t)) x(t)+F(r(\delta(t))) G(r(\delta(t))) x(\delta(t))] d t \\
& +\sum_{k=1}^{m} B_{k}(r(t)) x(t) d w_{k}(t) .
\end{aligned}
$$

It is observed that equation (2.4) is in fact a stochastic differential delay equation (SDDE) with a bounded variable delay (see e.g. [1]). So equation (2.4) has a unique solution $x(t)$ such that $\mathbb{E}|x(t)|^{2}<\infty$ for all $t \geq 0$ (see e.g. [17]).

\section{Stabilization of linear hybrid SDEs}

We will first denote $F(r(\delta(t))) G(r(\delta(t)))=$ $D(r(\delta(t)))$ and discuss the stability of the following hybrid stochastic system

$$
\begin{aligned}
d x(t) & =[A(r(t)) x(t)+D(r(\delta(t))) x(\delta(t))] d t \\
& +\sum_{k=1}^{m} B_{k}(r(t)) x(t) d w_{k}(t)
\end{aligned}
$$

in this section. And then design either $G(\cdot)$ given $F(\cdot)$ or $F(\cdot)$ given $G(\cdot)$ in order for the controlled SDE (2.4) to be stable.

Let us first give two lemmas for preparation.

Lemma 3.1 Let $x(t)$ be the solution of system (3.1). Set

$$
\begin{gathered}
M_{A}=\max _{i \in S}\left\|A_{i}\right\|^{2}, \quad M_{D}=\max _{i \in S}\left\|D_{i}\right\|^{2}, \\
M_{B}=\max _{i \in S} \sum_{k=1}^{m}\left\|B_{k i}\right\|^{2}
\end{gathered}
$$

and define

$$
K(\tau)=\left[6 \tau\left(\tau M_{A}+M_{B}\right)+3 \tau^{2} M_{D}\right] e^{6 \tau\left(\tau M_{A}+M_{B}\right)}
$$

for $\tau>0$. If $\tau$ is small enough for $2 K(\tau)<1$, then for any $t \geq 0$,

$$
\mathbb{E}|x(t)-x(\delta(t))|^{2} \leq \frac{2 K(\tau)}{1-2 K(\tau)} \mathbb{E}|x(t)|^{2} .
$$

Proof. Fix any integer $v \geq 0$. For $t \in[v \tau,(v+1) \tau)$, we have $\delta(t)=v \tau$. It follows from (3.1) that

$$
\begin{aligned}
x(t)-x(\delta(t)) & =x(t)-x(v \tau) \\
& =\int_{v \tau}^{t}[A(r(s)) x(s)+D(r(v \tau)) x(v \tau)] d s \\
& +\sum_{k=1}^{m} \int_{v \tau}^{t} B_{k}(r(s)) x(s) d w_{k}(s) .
\end{aligned}
$$

Using the fundamental inequality $|a+b+c|^{2} \leq$ $3|a|^{2}+3|b|^{2}+3|c|^{2}$ as well as Hölder's inequality and Doob's martingale inequality, we can then derive

$$
\begin{aligned}
& \mathbb{E}|x(t)-x(\delta(t))|^{2} \\
\leq & 3\left(\tau M_{A}+M_{B}\right) \int_{v \tau}^{t} \mathbb{E}|x(s)|^{2} d s \\
+ & 3 \tau^{2} M_{D} \mathbb{E}|x(v \tau)|^{2} \\
\leq & 6\left(\tau M_{A}+M_{B}\right) \int_{v \tau}^{t} \mathbb{E}|x(s)-x(\delta(s))|^{2} d s \\
+ & {\left[6 \tau\left(\tau M_{A}+M_{B}\right)+3 \tau^{2} M_{D}\right] \mathbb{E}|x(v \tau)|^{2} . }
\end{aligned}
$$

By the well-known Gronwall inequality, we have

$$
\mathbb{E}|x(t)-x(\delta(t))|^{2} \leq K(\tau) \mathbb{E}|x(v \tau)|^{2} .
$$

Consequently

$$
\begin{aligned}
& \mathbb{E}|x(t)-x(\delta(t))|^{2} \\
& \leq 2 K(\tau)\left(\mathbb{E}|x(t)-x(\delta(t))|^{2}+\mathbb{E}|x(t)|^{2}\right) .
\end{aligned}
$$

This implies that (3.3) holds for $t \in[v \tau,(v+1) \tau)$. But $v \geq 0$ is arbitrary, so the desired assertion (3.3) must hold for all $t \geq 0$. The proof is complete.

Lemma 3.2 For any $t \geq 0, v>0$ and $i \in S$,

$$
\begin{aligned}
& \mathbb{P}(r(s) \neq i \text { for some } s \in[t, t+v] \mid r(t)=i) \\
& \leq 1-e^{-\bar{\gamma} v}
\end{aligned}
$$

where

$$
\bar{\gamma}=\max _{i \in S}\left(-\gamma_{i i}\right)
$$


Proof. Given $r(t)=i$, define the stopping time

$$
\rho_{i}=\inf \{s \geq t: r(s) \neq i\},
$$

where and throughout this paper we set $\inf \emptyset=\infty$ (in which $\emptyset$ denotes the empty set as usual). It is well known (see e.g. [17]) that $\rho_{i}-t$ has the exponential distribution with parameter $-\gamma_{i i}$. Hence

$$
\begin{aligned}
& \mathbb{P}(r(s) \neq i \text { for some } s \in[t, t+v] \mid r(t)=i) \\
= & \mathbb{P}\left(\rho_{i}-t \leq v \mid r(t)=i\right)=\int_{0}^{v} \frac{1}{-\gamma_{i i}} e^{\gamma_{i i} s} d s \\
= & 1-e^{\gamma_{i i} v} \leq 1-e^{-\bar{\gamma} v}
\end{aligned}
$$

as desired.

We now state the main result on the exponential stability in mean-square of system (3.1).

Theorem 3.3 If there exist positive definite symmetric matrices $Q(i)=Q_{i}, i \in S$, such that

$$
\begin{aligned}
\bar{Q}(i)=\bar{Q}_{i}: & =Q_{i}\left(A_{i}+D_{i}\right)+\left(A_{i}+D_{i}\right)^{T} Q_{i} \\
& +\sum_{k=1}^{m} B_{k i}^{T} Q_{i} B_{k i}+\sum_{j=1}^{N} \gamma_{i j} Q_{j}
\end{aligned}
$$

are all negative-definite matrices. Set

$$
\begin{gathered}
M_{Q D}=\max _{i \in S}\left\|Q_{i} D_{i}\right\|^{2}, \quad N_{D}=\max _{i, j \in S}\left\|D_{j}-D_{i}\right\|^{2} \\
\text { and }-\lambda:=\max _{i \in S} \lambda_{\max }\left(\bar{Q}_{i}\right)
\end{gathered}
$$

(of course $\lambda>0$ ). If $\tau$ is sufficiently small for $\lambda>$ $2 \lambda_{\tau}+2 \lambda_{M} \mu_{\tau}$, where

$$
\lambda_{\tau}:=\sqrt{\frac{2 M_{Q D} K(\tau)}{1-2 K(\tau)}}, \quad \mu_{\tau}:=\sqrt{\frac{2 N_{D}\left(1-e^{-\bar{\gamma} \tau}\right)}{1-2 K(\tau)}},
$$

then the solution of the SDE (3.1) satisfies

$$
\mathbb{E}|x(t)|^{2} \leq \frac{\lambda_{M}}{\lambda_{m}} \mathbb{E}\left|x_{0}\right|^{2} e^{-\theta t}, \quad \forall t \geq 0,
$$

where $K(\tau)$ has been defined in Lemma 3.1 and

$$
\begin{gathered}
\lambda_{M}=\max _{i \in S} \lambda_{\max }\left(Q_{i}\right), \lambda_{m}=\min _{i \in S} \lambda_{\min }\left(Q_{i}\right), \\
\theta=\frac{\lambda-2 \lambda_{\tau}-2 \lambda_{M} \mu_{\tau}}{\lambda_{M}} .
\end{gathered}
$$

In other words, the SDE (3.1) is exponentially stable in mean square.
Proof. Let $V(x(t), r(t))=x^{T}(t) Q(r(t)) x(t)$. Applying the generalized Itô formula (see e.g. [17]) to $V$, we get

$$
d V(x(t), r(t))=\mathcal{L} V(x(t), r(t)) d t+d M_{1}(t),
$$

where $M_{1}(t)$ is a martingale with $M_{1}(0)=0$ and

$$
\begin{aligned}
& \mathcal{L} V(x(t), r(t)) \\
= & 2 x^{T}(t) Q(r(t))[A(r(t)) x(t)+D(r(\delta(t))) x(\delta(t))] \\
+ & \sum_{k=1}^{m} x^{T}(t) B_{k}^{T}(r(t)) Q(r(t)) B_{k}(r(t)) x(t) \\
+ & \sum_{j=1}^{N} \gamma_{r(t), j} x^{T}(t) Q_{j} x(t) \\
= & x^{T}(t) \bar{Q}(r(t)) x(t) \\
- & 2 x^{T}(t) Q(r(t)) D(r(t))(x(t)-x(\delta(t))) \\
- & 2 x^{T}(t) Q(r(t))(D(r(t))-D(r(\delta(t)))) x(\delta(t)) \\
\leq & -\lambda|x(t)|^{2}+2 \sqrt{M_{Q D}}|x(t)||x(t)-x(\delta(t))| \\
- & 2 x^{T}(t) Q(r(t))(D(r(t))-D(r(\delta(t)))) x(\delta(t))
\end{aligned}
$$

Applying the generalized Itô formula now to $e^{\theta t} x^{T}(t) Q(r(t)) x(t)$, we then have

$$
\begin{aligned}
& e^{\theta t} x^{T}(t) Q(r(t)) x(t)=x^{T}(0) Q(r(0)) x(0) \\
+ & \int_{0}^{t} e^{\theta s}\left[\theta x^{T}(s) Q(r(s)) x(s)+\mathcal{L} V(x(s), r(s))\right] d s \\
+ & M_{2}(t),
\end{aligned}
$$

where $M_{2}(t)$ is also a martingale with $M_{2}(0)=0$. Combining this with (3.10) yields

$$
\begin{aligned}
& \lambda_{m} e^{\theta t} \mathbb{E}|x(t)|^{2} \\
\leq & E\left(e^{\theta t} x^{T}(t) Q(r(t)) x(t)\right) \\
\leq & \lambda_{M} \mathbb{E}\left|x_{0}\right|^{2}+\int_{0}^{t}\left(\theta \lambda_{M}-\lambda\right) e^{\theta s} \mathbb{E}|x(s)|^{2} d s \\
+ & \int_{0}^{t} 2 e^{\theta s} \sqrt{M_{Q D}} \mathbb{E}(|x(s)||x(s)-x(\delta(s))|) d s \\
- & \int_{0}^{t} 2 e^{\theta s} \mathbb{E}\left(x^{T}(s) Q(r(s))(D(r(s))\right. \\
- & D(r(\delta(s)))) x(\delta(s))) d s .
\end{aligned}
$$


But, by Lemma 3.1 and 3.2, we have

$-2 e^{\theta s} \mathbb{E}\left(x^{T}(s) Q(r(s))(D(r(s))-D(r(\delta(s)))) x(\delta(s))\right)$

$\leq e^{\theta s} \mathbb{E}\left(\lambda_{M} \mu_{\tau}|x(s)|^{2}\right.$

$\left.+\frac{\lambda_{M}}{\mu_{\tau}}\|D(r(s))-D(r(\delta(s)))\|^{2}|x(\delta(s))|^{2}\right)$

$=e^{\theta s} \lambda_{M}\left\{\mu_{\tau} \mathbb{E}|x(s)|^{2}\right.$

$\left.+\frac{1}{\mu_{\tau}} \mathbb{E}\left(\mathbb{E}\left(\|D(r(s))-D(r(\delta(s)))\|^{2}|x(\delta(s))|^{2} \mid \mathcal{F}_{\delta(s)}\right)\right)\right\}$

$\leq e^{\theta s} \lambda_{M}\left\{\mu_{\tau} \mathbb{E}|x(s)|^{2}\right.$

$\left.+\frac{1}{\mu_{\tau}} \mathbb{E}\left(|x(\delta(s))|^{2} \sum_{r(\delta(s))=i} \mathcal{I}_{\{r(\delta(s))=i\}} \max _{i, j \in S}\left\|D_{j}-D_{i}\right\|^{2}\right)\right\}$

$\leq e^{\theta s} \lambda_{M}\left\{\mu_{\tau} \mathbb{E}|x(s)|^{2}+\frac{N_{D}\left(1-e^{-\bar{\gamma} \tau}\right)}{\mu_{\tau}} \mathbb{E}|x(\delta(s))|^{2}\right\}$

$\leq e^{\theta s} \lambda_{M}\left\{\mu_{\tau} \mathbb{E}|x(s)|^{2}\right.$

$\left.\left.+\frac{N_{D}\left(1-e^{-\bar{\gamma} \tau}\right)}{\mu_{\tau}} \frac{2}{1-2 K(\tau)} \mathbb{E}|x(s)|^{2}\right)\right\}$

$=2 e^{\theta s} \lambda_{M} \mu_{\tau} \mathbb{E}|x(s)|^{2}$

and

$$
\begin{aligned}
& 2 \sqrt{M_{Q D}} \mathbb{E}(|x(s)||x(s)-x(\delta(s))|) \\
\leq & \lambda_{\tau} \mathbb{E}|x(s)|^{2}+\frac{M_{Q D}}{\lambda_{\tau}} \mathbb{E}|x(s)-x(\delta(s))|^{2} \\
\leq & \lambda_{\tau} \mathbb{E}|x(s)|^{2}+\frac{M_{Q D}}{\lambda_{\tau}} \frac{2 K(\tau)}{1-2 K(\tau)} \mathbb{E}|x(s)|^{2} \\
= & 2 \lambda_{\tau} \mathbb{E}|x(s)|^{2}
\end{aligned}
$$

Substituting (3.12)(3.13) into (3.11) gives

$$
\begin{aligned}
& \lambda_{m} e^{\theta t} \mathbb{E}|x(t)|^{2} \leq \lambda_{M} \mathbb{E}\left|x_{0}\right|^{2} \\
+ & \int_{0}^{t}\left(\theta \lambda_{M}+2 \lambda_{\tau}+2 \lambda_{M} \mu_{\tau}-\lambda\right) e^{\theta s} \mathbb{E}|x(s)|^{2} d s .
\end{aligned}
$$

But, by (3.9), $\theta \lambda_{M}+2 \lambda_{\tau}+2 \lambda_{M} \mu_{\tau}-\lambda=0$. Thus

$$
\lambda_{m} e^{\theta t} \mathbb{E}|x(t)|^{2} \leq \lambda_{M} \mathbb{E}\left|x_{0}\right|^{2},
$$

which implies the desired assertion (3.8). The proof is complete.

The following two corollaries provide us with an LMI method to design the controller based on discrete-time observations of both state and mode to stabilize the unstable system (2.1). Corollary 3.4 and 3.5 demonstrate the case of state feedback and output injection, respectively.
Corollary 3.4 Assume that there are solutions $Q_{i}=$ $Q_{i}^{T}>0$ and $Y_{i}(i \in S)$ to the following LMIs

$$
\begin{gathered}
Q_{i} A_{i}+Y_{i} G_{i}+A_{i}^{T} Q_{i}+G_{i}^{T} Y_{i}^{T} \\
+\sum_{k=1}^{m} B_{k i}^{T} Q_{i} B_{k i}+\sum_{j=1}^{N} \gamma_{i j} Q_{j}<0 .
\end{gathered}
$$

Then by setting $F_{i}=Q_{i}^{-1} Y_{i}$ and $D_{i}=F_{i} G_{i}$, the controlled SDE (2.4) will be exponentially stable in mean square if $\tau>0$ is sufficiently small for $\lambda>2 \lambda_{\tau}+$ $2 \lambda_{M} \mu_{\tau}$.

Proof. Recalling $F_{i}=Q_{i}^{-1} Y_{i}$ and $D_{i}=F_{i} G_{i}$, we find that (3.14) is equivalent to the condition that matrices in (3.6) are all negative-definite. So the required assertion follows directly from Theorem 3.3.

Corollary 3.5 Assume that there are solutions $X_{i}=$ $X_{i}^{T}>0$ and $Y_{i}(i \in S)$ to the following LMIs

$$
\left[\begin{array}{ccc}
M_{i 1} & M_{i 2} & M_{i 3} \\
M_{i 2}^{T} & -M_{i 4} & 0 \\
M_{i 3}^{T} & 0 & -M_{i 5}
\end{array}\right]<0,
$$

where

$$
\begin{aligned}
& M_{i 1}=A_{i} X_{i}+F_{i} Y_{i}+X_{i} A_{i}^{T}+Y_{i}^{T} F_{i}^{T}+\gamma_{i i} X_{i}, \\
& M_{i 2}=\left[X_{i} B_{1 i}^{T}, \cdots, X_{i} B_{m i}^{T}\right], \\
& M_{i 3}=\left[\sqrt{\gamma_{i 1}} X_{i}, \cdots, \sqrt{\gamma_{i(i-1)}} X_{i}, \sqrt{\gamma_{i(i+1)}} X_{i}, \cdots, \sqrt{\gamma_{i N}} X_{i}\right], \\
& M_{i 4}=\operatorname{diag}\left[X_{i}, \cdots, X_{i}\right], \\
& M_{i 5}=\operatorname{diag}\left[X_{1}, \cdots, X_{i-1}, X_{i+1}, \cdots, X_{N}\right] .
\end{aligned}
$$

Then by setting $Q_{i}=X_{i}^{-1}, G_{i}=Y_{i} X_{i}^{-1}$ and $D_{i}=$ $F_{i} G_{i}$, the controlled $S D E$ (2.4) will be exponentially stable in mean square if $\tau>0$ is sufficiently small for $\lambda>2 \lambda_{\tau}+2 \lambda_{M} \mu_{\tau}$.

Proof. We first observe that by the well-known Schur complements (see e.g. [17]), the LMIs (3.15) are equivalent to the following matrix inequalities

$$
\begin{aligned}
& A_{i} X_{i}+F_{i} Y_{i}+X_{i} A_{i}^{T}+Y_{i}^{T} F_{i}^{T}+\gamma_{i i} X_{i} \\
& +\sum_{k=1}^{m} X_{i} B_{k i}^{T} X_{i}^{-1} B_{k i} X_{i}+\sum_{j \neq i}^{N} \gamma_{i j} X_{i} X_{j}^{-1} X_{i}<0 .
\end{aligned}
$$

Recalling that $G_{i}=Y_{i} X_{i}^{-1}$ and $X_{i}=X_{i}^{T}$, we have

$$
\begin{aligned}
& A_{i} X_{i}+F_{i} G_{i} X_{i}+X_{i} A_{i}^{T}+X_{i} G_{i}^{T} F_{i}^{T} \\
& +\sum_{k=1}^{m} X_{i} B_{k i}^{T} X_{i}^{-1} B_{k i} X_{i}+\sum_{j=1}^{N} \gamma_{i j} X_{i} X_{j}^{-1} X_{i}<0 .
\end{aligned}
$$


Multiplying $X_{i}^{-1}$ from left and then from right, and noting $Q_{i}=X_{i}^{-1}, D_{i}=F_{i} G_{i}$, we see that the matrix inequalities (3.18) are equivalent to the following matrix inequalities

$$
\begin{gathered}
Q_{i} A_{i}+Q_{i} D_{i}+A_{i}^{T} Q_{i}+D_{i}^{T} Q_{i}+ \\
\sum_{k=1}^{m} B_{k i}^{T} Q_{i} B_{k i}+\sum_{j=1}^{N} \gamma_{i j} Q_{j}<0
\end{gathered}
$$

which yields matrices in (3.6) are all negative-definite. Again, the required assertion follows directly from Theorem 3.3.

\section{Stabilization of nonlinear hybrid SDEs}

Let us now develop our theory to cope with the more general nonlinear stabilization problem. For an unstable nonlinear hybrid SDE

$$
d x(t)=f(x(t), r(t), t) d t+g(x(t), r(t), t) d w(t)
$$

on $t \geq 0 \quad$ with the initial data $x(0)=x_{0} \in$ $L_{\mathcal{F}_{0}}^{2}\left(R^{n}\right) . \quad$ Here, $\quad f: R^{n} \times S \times R_{+} \rightarrow R^{n} \quad$ and $g: R^{n} \times S \times R_{+} \rightarrow R^{n \times m}$. Assume that both $f$ and $g$ are globally Lipschitz continuous and hence obey the linear growth condition (see e.g. [17]).

Assumption 4.1 Assume that the coefficients $f$ and $g$ are globally Lipschitz continuous (see e.g. [7, 8, 9, 17]). That is, we have

$$
\begin{array}{r}
|f(x, i, t)-f(y, i, t)| \leq K_{1}|x-y| \\
\text { and } \quad|g(x, i, t)-g(y, i, t)| \leq K_{2}|x-y|,
\end{array}
$$

for all $(x, i, t),(y, i, t) \in R^{n} \times S \times R_{+}$, where both $K_{1}$ and $K_{2}$ are positive numbers.

We also assume that $f(0, i, t)=0$ and $g(0, i, t)=0$ for all $i \in S$ and $t \geq 0$ so that $x=0$ is an equilibrium point for (4.1).

Hence, $f, g$ satisfy the following linear growth condition as stated in Assumption 4.3 with $\delta_{1}=K_{1}^{2}$ and $\delta_{2}=K_{2}^{2}$.

We are required to design a linear feedback control $F(r(t)) G(r(t)) x(\delta(t))$ based on the discrete-time state and mode observations in the drift part so that the controlled system

$$
\begin{aligned}
d x(t) & =[f(x(t), r(t), t)+F(r(t)) G(r(t)) x(\delta(t))] d t \\
& +g(x(t), r(t), t) d w(t)
\end{aligned}
$$

will be mean-square exponentially stable. Defining $\zeta$ : $[0, \infty) \rightarrow[0, \tau]$ by

$$
\zeta(t)=t-v \tau \quad \text { for } \quad v \tau \leq t<t(v+1) \tau,
$$

and $v=0,1,2, \cdots$, then we see that the $\operatorname{SDE}$ (4.3) can be written as an SDDE

$$
\begin{aligned}
& d x(t)=[f(x(t), r(t), t)+ \\
& \quad F(r(t-\zeta(t))) G(r(t-\zeta(t))) x(t-\zeta(t))] d t \\
& \quad+g(x(t), r(t), t) d w(t) .
\end{aligned}
$$

It is therefore known (see e.g. [17]) that equation (4.3) has a unique solution $x(t)$ such that $\mathbb{E}|x(t)|^{2}<\infty$ for all $t \geq 0$.

In order to stabilize a nonlinear system by a linear control, we impose some conditions on the nonlinear coefficients $f$ and $g$ as follows.

Assumption 4.2 For each $i \in S$, there is a pair of symmetric $n \times n$-matrices $Q_{i}$ and $\hat{Q}_{i}$ with $Q_{i}$ being positive-definite such that

$$
2 x^{T} Q_{i} f(x, i, t)+g^{T}(x, i, t) Q_{i} g(x, i, t) \leq x^{T} \hat{Q}_{i} x
$$

for all $(x, i, t) \in R^{n} \times S \times R_{+}$.

Assumption 4.3 There is a pair of positive constants $\delta_{1}$ and $\delta_{2}$ such that

$$
|f(x, i, t)|^{2} \leq \delta_{1}|x|^{2} \quad \text { and } \quad|g(x, i, t)|^{2} \leq \delta_{2}|x|^{2}
$$

for all $(x, i, t) \in R^{n} \times S \times R_{+}$.

Let us first present a useful lemma.

Lemma 4.4 Let Assumption 4.3 hold. Set

$$
\delta_{3}=\max _{i \in S} \sum_{k=1}^{m}\left\|F_{i} G_{i}\right\|^{2},
$$

and define

$$
H(\tau)=\left[6 \tau\left(\tau \delta_{1}+\delta_{2}\right)+3 \tau^{2} \delta_{3}\right] e^{6 \tau\left(\tau \delta_{1}+\delta_{2}\right)}
$$

for $\tau>0$. If $\tau$ is sufficiently small for $2 H(\tau)<1$, then the solution $x(t)$ of the SDE (4.3) satisfies

$$
\mathbb{E}|x(t)-x(\delta(t))|^{2} \leq \frac{2 H(\tau)}{1-2 H(\tau)} \mathbb{E}|x(t)|^{2}
$$

for all $t \geq 0$.

This lemma can be proved in the same way as Lemma 3.1 was proved so we omit the proof. 
Theorem 4.5 Let Assumptions 4.2 and 4.3 hold. Assume that the following LMIs

$$
\begin{aligned}
U_{i}: & =\hat{Q}_{i}+Q_{i} F_{i} G_{i}+G_{i}^{T} F_{i}^{T} Q_{i} \\
& +\sum_{j=1}^{N} \gamma_{i j} Q_{j}<0, \quad i \in S,
\end{aligned}
$$

have their solutions $F_{i}(i \in S)$ in the case of feedback control (i.e. $G_{i}$ 's are given), or their solutions $G_{i}$ in the case of output injection (i.e. $F_{i}$ 's are given). Set

$$
\begin{gathered}
-\gamma:=\max _{i \in S} \lambda_{\max }\left(U_{i}\right) \quad \text { and } \quad \delta_{4}=\max _{i \in S}\left\|Q_{i} F_{i} G_{i}\right\|^{2}, \\
\delta_{5}=\max _{i, j \in S}\left\|F_{i} G_{i}-F_{j} G_{j}\right\|^{2} .
\end{gathered}
$$

If $\tau$ is sufficiently small for $\gamma>2 \gamma_{\tau}+2 \lambda_{M} \eta_{\tau}$, where

$$
\gamma_{\tau}:=\sqrt{\frac{2 \delta_{4} H(\tau)}{1-2 H(\tau)}}, \quad \eta_{\tau}:=\sqrt{\frac{2 \delta_{5}\left(1-e^{-\bar{\gamma} \tau}\right)}{1-2 H(\tau)}}
$$

then the solution of the SDE (4.3) satisfies

$$
\mathbb{E}|x(t)|^{2} \leq \frac{\lambda_{M}}{\lambda_{m}} \mathbb{E}\left|x_{0}\right|^{2} e^{-\theta t}, \quad \forall t \geq 0,
$$

where $H(\tau)$ has been defined in Lemma 4.4 and

$$
\begin{gathered}
\lambda_{M}=\max _{i \in S} \lambda_{\max }\left(Q_{i}\right), \quad \lambda_{m}=\min _{i \in S} \lambda_{\min }\left(Q_{i}\right), \\
\theta=\frac{\gamma-2 \gamma_{\tau}-2 \lambda_{M} \eta_{\tau}}{\lambda_{M}} .
\end{gathered}
$$

Proof. This theorem can be proved in a similar way as Theorem 3.3 was proved so we only give the key steps. Applying the generalized Itô formula to $x^{T}(t) Q(r(t)) x(t)$ we get

$$
\begin{aligned}
& d\left[x^{T}(t) Q(r(t)) x(t)\right] \\
= & \left(x^{T}(t) U(r(t)) x(t)\right. \\
- & 2 x^{T}(t) Q(r(t)) F(r(t)) G(r(t))(x(t)-x(\delta(t))) \\
- & 2 x^{T}(t) Q(r(t)) \\
& F(r(t)-r(\delta(t))) G(r(t)-r(\delta(t))) x(\delta(t))) d t \\
+ & d M_{3}(t),
\end{aligned}
$$

where $M_{3}(t)$ is a martingale with $M_{3}(0)=0$. Applying the generalized Itô formula further to $e^{\theta t} x^{T}(t) Q(r(t)) x(t)$, we can then obtain

$$
\begin{aligned}
& \lambda_{m} e^{\theta t} \mathbb{E}|x(t)|^{2} \\
\leq & \lambda_{M} \mathbb{E}\left|x_{0}\right|^{2}+\int_{0}^{t}\left(\theta \lambda_{M}-\gamma\right) e^{\theta s} \mathbb{E}|x(s)|^{2} d s \\
+ & \int_{0}^{t} 2 e^{\theta s} \sqrt{\delta_{4}} \mathbb{E}(|x(s)||x(s)-x(\delta(s))|) d s \\
+ & \int_{0}^{t} 2 \mathbb{E}\left(e^{\theta s} x^{T}(s) Q(r(s))(F(r(s)) G(r(s))\right. \\
- & F(r(\delta(s))) G(r(\delta(s)))) x(\delta(s))) d s .
\end{aligned}
$$

But, by Lemma 4.4, we can show

$$
2 \sqrt{\delta_{4}} \mathbb{E}(|x(s)||x(s)-x(\delta(s))|) \leq 2 \gamma_{\tau} \mathbb{E}|x(s)|^{2},
$$

while by Lemma 3.2 and (4.9) we can prove that

$$
\begin{aligned}
& 2 \mathbb{E}\left(e^{\theta s} x^{T}(s) Q(r(s))(F(r(s)) G(r(s))\right. \\
- & F(r(\delta(s))) G(r(\delta(s)))) x(\delta(s))) \\
\leq & 2 e^{\theta s} \lambda_{M} \eta_{\tau} \mathbb{E}|x(s)|^{2} .
\end{aligned}
$$

Substituting this into (4.12) yields

$$
\lambda_{m} e^{\theta t} \mathbb{E}|x(t)|^{2} \leq \lambda_{M} \mathbb{E}\left|x_{0}\right|^{2},
$$

which implies the desired assertion (4.10). The proof is complete.

To apply Theorem 4.5, we need two steps:

1 we first need to look for the $2 N$ matrices $Q_{i}$ and $\hat{Q}_{i}$ for Assumption 4.2 to hold;

2 we then need to solve the LMIs in (4.8) for their solutions $F_{i}$ (or $\left.G_{i}\right)$.

There are available computer softwares e.g. Matlab for step 2 so in the remaining part of this section we will develop some ideas for step 1 . To make our ideas more clear, we will only consider the case of feedback control, but the same ideas work for the case of output injection.

In theory, it is flexible to use $2 N$ matrices $Q_{i}$ and $\hat{Q}_{i}$ in Assumption 4.2. But, in practice, it means more work to be done in finding these $2 N$ matrices. It is in this spirit that we introduce a stronger assumption.

Assumption 4.6 There are $N+1$ symmetric $n \times n$ matrices $Z$ and $Z_{i}(i \in S)$ with $Z>0$ such that

$$
2 x^{T} Z f(x, i, t)+g^{T}(x, i, t) Z g(x, i, t) \leq x^{T} Z_{i} x
$$

for all $(x, i, t) \in R^{n} \times S \times R_{+}$. 
Under this assumption, if we let $Q_{i}=q_{i} Z$ and $\hat{Q}_{i}=$ $q_{i} Z_{i}$ for some positive numbers $q_{i}$, then Assumption 4.2 holds. Moreover, the LMIs in (4.8) become

$$
\begin{gathered}
q_{i} Z_{i}+q_{i} Z F_{i} G_{i}+q_{i} G_{i}^{T} F_{i}^{T} Z \\
+\sum_{j=1}^{N} \gamma_{i j} q_{j} Z<0, \quad i \in S .
\end{gathered}
$$

If we set $Y_{i}:=q_{i} F_{i}$, then these become the following LMIs in $q_{i}$ and $Y_{i}$ :

$$
\begin{aligned}
& q_{i} Z_{i}+Z Y_{i} G_{i}+G_{i}^{T} Y_{i}^{T} Z \\
& +\sum_{j=1}^{N} \gamma_{i j} q_{j} Z<0, \quad i \in S .
\end{aligned}
$$

We hence have the following corollary.

Corollary 4.7 Let Assumptions 4.6 and 4.3 hold. Assume that the LMIs (4.15) have their solutions $q_{i}>$ 0 and $Y_{i}(i \in S)$. Then Theorem 4.5 holds by setting $Q_{i}=q_{i} Z, \hat{Q}_{i}=q_{i} Z_{i}$ and $F_{i}=q_{i}^{-1} Y_{i}$. In other words, the controlled $S D E$ (4.3) will be exponentially stable in mean square if we set $F_{i}=q_{i}^{-1} Y_{i}$ and make sure $\tau>0$ be sufficiently small for $\gamma>2 \gamma_{\tau}+2 \lambda_{M} \eta_{\tau}$.

An even simpler (but in fact stronger) condition is:

Assumption 4.8 There are constants $z_{i}(i \in S)$ such that

$$
2 x^{T} f(x, i, t)+|g(x, i, t)|^{2} \leq z_{i}|x|^{2}
$$

for all $(x, i, t) \in R^{n} \times S \times R_{+}$.

Under this assumption, if we let $Q_{i}=q_{i} I$ and $\hat{Q}_{i}=$ $q_{i} z_{i} I$ for some positive numbers $q_{i}$, where $I$ is the $n \times n$ identity matrix, then Assumption 4.2 holds. Moreover, the LMIs in (4.8) become

$$
\begin{aligned}
& q_{i} z_{i} I+q_{i} F_{i} G_{i}+q_{i} G_{i}^{T} F_{i}^{T} \\
& +\sum_{j=1}^{N} \gamma_{i j} q_{j} I<0, \quad i \in S .
\end{aligned}
$$

If we set $Y_{i}:=q_{i} F_{i}$, then these become the following LMIs in $q_{i}$ and $Y_{i}$ :

$$
\begin{aligned}
& q_{i} z_{i} I+Y_{i} G_{i}+G_{i}^{T} Y_{i}^{T} \\
& +\sum_{j=1}^{N} \gamma_{i j} q_{j} I<0, \quad i \in S .
\end{aligned}
$$

We hence have another corollary.
Corollary 4.9 Let Assumptions 4.8 and 4.3 hold. Assume that the LMIs (4.16) have their solutions $q_{i}>$ 0 and $Y_{i}(i \in S)$. Then Theorem 4.5 holds by setting $Q_{i}=q_{i} I, \hat{Q}_{i}=q_{i} z_{i} I$ and $F_{i}=q_{i}^{-1} Y_{i}$. In other words, the controlled SDE (4.3) will be exponentially stable in mean square if we set $F_{i}=q_{i}^{-1} Y_{i}$ and make sure $\tau>0$ be sufficiently small for $\gamma>2 \gamma_{\tau}+2 \lambda_{M} \eta_{\tau}$.

\section{Example}

Let us consider an unstable linear hybrid SDE

$$
d x(t)=A(r(t)) x(t) d t+B(r(t)) x(t) d w(t)
$$

on $t \geq t_{0}$. Here $w(t)$ is a scalar Brownian motion; $r(t)$ is a Markov chain on the state space $S=\{1,2\}$ with the generator

$$
\Gamma=\left[\begin{array}{rr}
-1 & 1 \\
1 & -1
\end{array}\right]
$$

and the system matrices are

$$
\begin{array}{ll}
A_{1}=\left[\begin{array}{rr}
1 & -1 \\
1 & -5
\end{array}\right], & A_{2}=\left[\begin{array}{rr}
-5 & -1 \\
1 & 1
\end{array}\right], \\
B_{1}=\left[\begin{array}{rr}
1 & 1 \\
1 & -1
\end{array}\right], & B_{2}=\left[\begin{array}{rr}
-1 & -1 \\
-1 & 1
\end{array}\right] .
\end{array}
$$

The computer simulation (Fig. 1) shows this hybrid $\mathrm{SDE}$ is not mean square exponentially stable.

Let us now design a discrete-time-state feedback control to stabilize the system. Assume that the controlled hybrid SDE has the form

$$
\begin{aligned}
d x(t) & =[A(r(t)) x(t)+F(r(\delta(t))) G(r(\delta(t))) x(\delta(t))] d t \\
& +B(r(t)) x(t) d w(t),
\end{aligned}
$$

where

$$
G_{1}=[1,0], \quad G_{2}=[0,1] .
$$

Our aim is to find $F_{1}$ and $F_{2}$ in $R^{2 \times 1}$ and then make sure $\tau$ is sufficiently small for this controlled SDE to be exponentially stable in mean square. To apply Corollary 3.4, we first find that the following LMIs

$$
\begin{aligned}
\bar{Q}_{i}:= & Q_{i} A_{i}+Y_{i} G_{i}+A_{i}^{T} Q_{i}+G_{i}^{T} Y_{i}^{T}+B_{i}^{T} Q_{i} B_{i} \\
& +\sum_{j=1}^{2} \gamma_{i j} Q_{j}<0, \quad i=1,2,
\end{aligned}
$$

have the following set of solutions

$$
Q_{1}=\left[\begin{array}{ll}
1 & 0 \\
0 & 2
\end{array}\right], \quad Q_{2}=\left[\begin{array}{ll}
2 & 0 \\
0 & 1
\end{array}\right],
$$



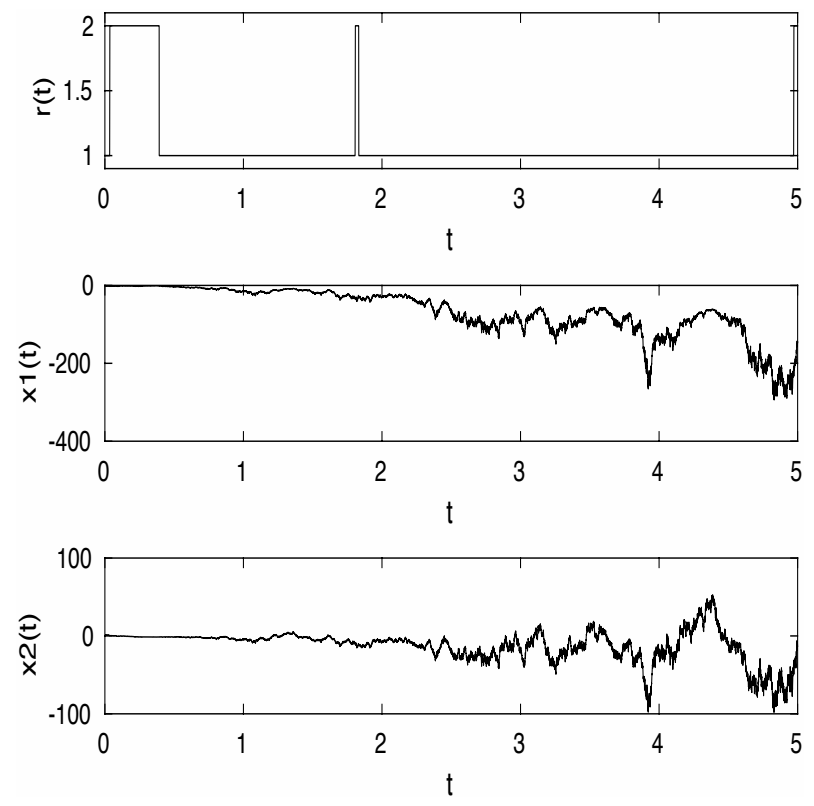

Fig. 1. Computer simulation of the paths of $r(t), x_{1}(t)$ and $x_{2}(t)$ for the hybrid SDE (5.1) using the Euler-Maruyama method with step size $10^{-6}$ and initial values $r(0)=1, x_{1}(0)=-2$ and $x_{2}(0)=1$.

and

$$
Y_{1}=\left[\begin{array}{c}
-10 \\
0
\end{array}\right], \quad Y_{2}=\left[\begin{array}{c}
0 \\
-10
\end{array}\right],
$$

and for these solutions we have

$$
\bar{Q}_{1}=\left[\begin{array}{rr}
-7 & 0 \\
0 & -1
\end{array}\right], \quad \bar{Q}_{2}=\left[\begin{array}{rr}
-1 & 0 \\
0 & -7
\end{array}\right] .
$$

Hence, we have

$-\lambda=\max _{i=1,2} \lambda_{\max }\left(\hat{Q}_{i}\right)=-1, M_{Y G}=\max _{i=1,2}\left\|Y_{i} G_{i}\right\|^{2}=100$.

It is easy to compute that

$M_{A}=27.42, M_{B}=2, M_{D}=100, M_{Q D}=100, N_{D}=100$

Hence

$$
\lambda_{\tau}=\sqrt{\frac{200 K(\tau)}{1-2 K(\tau)}}, \quad \mu_{\tau}=\sqrt{\frac{200\left(1-e^{-\bar{\gamma} \tau}\right)}{1-2 K(\tau)}}
$$

where $K(\tau)=\left[6 \tau(27.42 \tau+2)+300 \tau^{2}\right] e^{6 \tau(27.42 \tau+2)}$. By calculating, we get that $\lambda>2 \lambda_{\tau}+2 \lambda_{M} \mu_{\tau}$ whenever $\tau<0.000015$. By Corollary 3.4, if we set $F_{1}=$ $Y_{1}$ and $F_{2}=Y_{2}$, and make sure that $\tau<1.5 \times 10^{-5}$, then the discrete-time-state feedback controlled hybrid SDE (5.2) is mean-square exponentially stable. The computer simulation (Fig. 2) supports this result clearly.
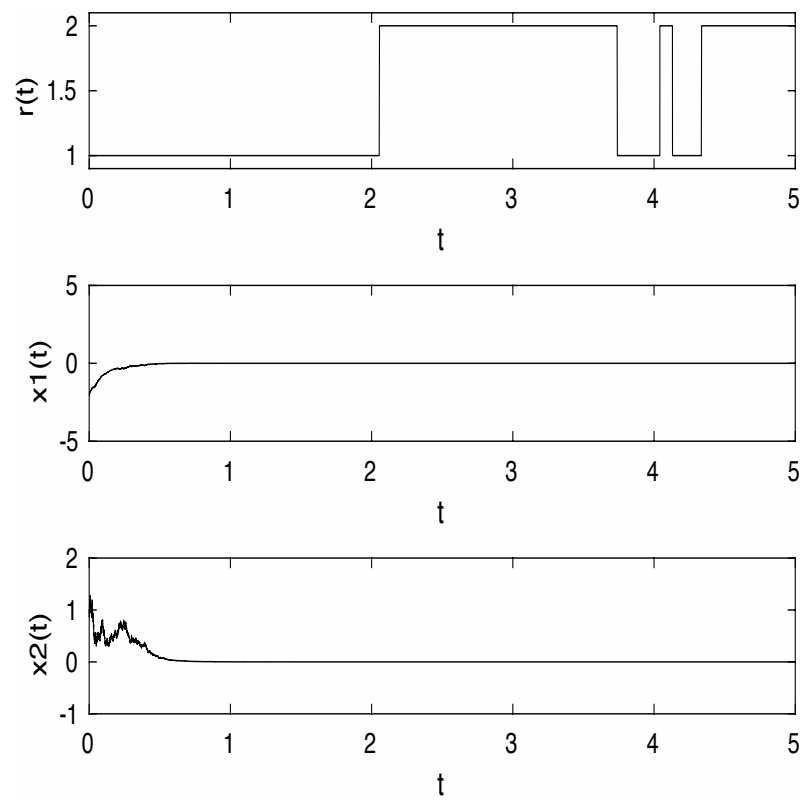

Fig. 2. Computer simulation of the paths of $r(t), x_{1}(t)$ and $x_{2}(t)$ for the controlled hybrid $\operatorname{SDE}$ (5.2) with $\tau=10^{-3}$ using the Euler-Maruyama method with step size $10^{-6}$ and initial values $r(0)=1, x_{1}(0)=-2$ and $x_{2}(0)=1$.

\section{Conclusion}

In this paper, we have proved that unstable linear hybrid SDEs, in the form of (2.1), can be stabilized by a feedback control based on discrete-time state and mode observations. Moreover, we have generalised the theory to a class of nonlinear systems.

\section{Acknowledgements}

The author wold like to thank the reviewers and the editor for their very helpful comments and professional suggestions. The authors would also like to thank the Leverhulme Trust (RF-2015-385), the Royal Society of London (IE131408), and the Ministry of Education (MOE) of China (MS2014DHDX020) for their financial support. In particular, the first author would like to thank the University of Strathclyde for awarding her the $\mathrm{PhD}$ studentship.

\section{REFERENCES}

1. Mao,X., Stabilization of continuous-time hybrid stochastic differential equations by discrete-time feedback control, Automatics 49(12) (2013), 3677-3681.

2. Basak, G.K., Bisi, A. and Ghosh, M.K., Stability of a random diffusion with linear drift, J. Math. Anal. Appl. 202 (1996), 604-622. 
3. Gao, H., Wang, C. and Wang, L., On $H_{\infty}$ performance analysis for continuous-time stochastic systems with polytopic uncertainties, Circuits Systems Signal Processing 24 (2005), 415-429.

4. He, H., Ho, D.W.C. and Lam, J., Stochastic stability analysis of fuzzy Hopfield neural networks with time-varying delays, IEEE Trans. Circuits and Systems II: Express Briefs 52 (2005), 251 255.

5. Hu, L., Mao, X., Almost sure exponential stabilization of stochastic systems by statefeedback control, Automatica 44 (2008), 465-471.

6. Ji, Y. and Chizeck, H.J., Controllability, stabilizability and continuous-time Markovian jump linear quadratic control, IEEE Trans. Automat. Control 35 (1990), 777-788.

7. Mao, X., Stability of Stochastic Differential Equations with Respect to Semimartingales, Longman Scientific and Technical, 1991.

8. Mao, X., Exponential Stability of Stochastic Differential Equations, Marcel Dekker, 1994.

9. Mao X., Stochastic Differential Equations and Their Applications, 2nd Edition, Chichester: Horwood Pub., 2007.

10. Mao, X., Stability of stochastic differential equations with Markovian switching, Sto. Proc. Their Appl. 79 (1999), 45-67.

11. Mao, X., Exponential stability of stochastic delay interval systems with Markovian switching, IEEE Trans. Auto. Control 47(10) (2002), 1604-1612.

12. Mao, X., Stability and stabilization of stochastic differential delay equations, IET Control Theory \& Applications 1(6) (2007), 1551-1566.

13. Mao, X., Matasov, A. and Piunovskiy, A.B., Stochastic differential delay equations with Markovian switching, Bernoulli 6(1) (2000), 73-90.

14. Mao, X., Yin, G. and Yuan, C., Stabilization and destabilization of hybrid systems of stochastic differential equations, Automatica 43 (2007), 264273.

15. Mao, X., Lam, J. and Huang, L., Stabilisation of hybrid stochastic differential equations by delay feedback control, Systems \& Control Letters 57 (2008), 927-935.

16. Mao, X., Liu, W., Hu, L., Luo, Q. and Lu, J., Stabilization of Hybrid SDEs by Feedback control based on discrete-time state observations, Systems and Control Letters 73 (2014), 88-95.

17. Mao, X. and Yuan, C., Stochastic Differential Equations with Markovian Switching, Imperial College Press, 2006.
18. Mariton, M., Jump Linear Systems in Automatic Control, Marcel Dekker, 1990.

19. Mohammed, S.-E.A., Stochastic Functional Differential Equations, Longman Scientific and Technical, 1986.

20. Niu, Y., Ho, D.W.C. and Lam, J., Robust integral sliding mode control for uncertain stochastic systems with time-varying delay, Automatica 41 (2005), 873-880.

21. Shaikhet, L., Stability of stochastic hereditary systems with Markov switching, Theory of Stochastic Processes 2(18) (1996), 180-184.

22. Shi, P., Mahmoud, M.S., Yi, J. and Ismail, A., Worst case control of uncertain jumping systems with multi-state and input delay information, Information Sciences 176 (2006), 186-200.

23. Sun, M., Lam, J., Xu, S. and Zou, Y., Robust exponential stabilization for Markovian jump systems with mode-dependent input delay, Automatica 43 (2007), 1799-1807.

24. Willsky, A.S. and Levy,B.C., Stochastic stability research for complex power systems, DOE Contract, LIDS, MIT, Report ET-76-C-01-2295, 1979.

25. Wang, Z., Qiao, H., Burnham, K.J., On stabilization of bilinear uncertain time-delay stochastic systems with Markovian jumping parameters, IEEE Trans. Automat. Control 47(4) (2002), 640646.

26. Wu, L., Shi, P. and Gao, H., State estimation and sliding mode control of Markovian jump singular systems, IEEE Trans on Automatic Control 55(5) (2010), 1213-1219.

27. Wu, L., Su, X. and Shi, P., Sliding mode control with bounded $L_{2}$ gain performance of Markovian jump singular time-delay systems, Automatica 48(8) (2012), 1929-1933.

28. Wei, G., Wang, Z., Shu, H. and Fang, J., Robust $H_{\infty}$ control of stochastic time-delay jumping systems with nonlinear disturbances, Optim. Control Appl. Meth. 27 (2006), 255-271.

29. Xu, S., Lam, J., Wang J.L. and Yang, G.H., Stabilization and $H_{\infty}$ control for uncertain stochastic time-delay systems via non-fragile controllers, Asian Journal of Control 8 (2006), 197-200.

30. Yue, D. and Han, Q., Delay-dependent exponential stability of stochastic systems with time-varying delay, nonlinearity, and Markovian switching, IEEE Trans. Automat. Control 50 (2005), 217222. 